\title{
Modelling the Role of Trail Pheromone in the Collective Construction of Termite Royal Chambers
}

\author{
Nicholas Hill ${ }^{1}$ and Seth Bullock \\ Institute for Complex Systems Simulation, University of Southampton, UK, SO17 1BJ \\ ${ }^{1}$ n.c.hillesoton.ac.uk
}

\begin{abstract}
Experiments with worker termites constructing a royal chamber around a termite queen in species Macrotermes subhyalinus (Rambur) have shown that both trail and cement pheromones are involved and necessary for the successful formation of pillars during the building process. However, earlier models of the construction were able to demonstrate stigmergic pillar formation with cement pheromone alone. We present results from a new three-dimensional agent-based model, developed to investigate the role of trail pheromone in the construction process. The model is able to demonstrate how, if the properties of the cement pheromone are altered so that its attractive influence is more localised than in earlier models, termites are unable to produce significant pillar formation. The model shows how the addition of trail deposition and following effectively increases the range of the stigmergic effect so that pillar formation is restored. The presence of trail pheromone also results in pillars which are narrower than those produced by cement pheromone alone, and which show more pronounced lateral extensions. Additionally the paths that the termites take from the termite queen to building sites become more directed with time. These features are in keeping with observation and have not been previously modelled.
\end{abstract}

\section{Introduction}

As a scientific field, artificial life has studied the natural processes through which collections of simple components can give rise to complex systemic behaviour. One example of such a collective process is morphogenesis, the development of coherent structure in biological systems. Colonies of social insects demonstrate this process when, for example, a collective effort is required in order to construct a nest or when the individuals in a colony coalesce to form useful structures, such as rafts (Wilson, 1971).

Morphogenetic engineering is the study of the ways in which artificial morphogenetic systems can be created and directed to ensure the formation of structures with prespecified requirements (Doursat et al., 2012). Understanding the dynamics of social insect construction may enable the extraction of design principles which facilitate the creation of teams of agents that are capable of building functional structures in remote and hostile environments, in an environmentally adaptive, self-organised and self repairing manner.

\section{Termites and Collective Construction}

Some species of termite are able to construct nests in the form of mounds that are orders of magnitude greater in size than the individual termites and which have several functionally distinct sections (Howse, 1970; Wilson, 1971). There is no evidence, however, that these termites rely on centralised blueprints or engage in planning, deliberation or negotiation during mound construction. Instead they act independently and instinctively, responding to multiple local cues in their immediate environment, including air flow, humidity and temperature, and also to the presence of any existing built structures (Grassé, 1959; Stuart, 1967; Howse, 1970). The ways in which combinations of environmental signals and instinctive responses enable termites to self-organise their building effort are not well understood. There is evidence, however, that the trail pheromone that they synthesize and deposit acts as a chemical signal diffusing through the environment and is a key factor in many aspects of termite behaviour (Stuart, 1970; Leuthold et al., 1976). Accordingly, here we develop a model of an early stage of termite nest construction in order to investigate how trail pheromone may be of central importance.

In species Macrotermes subhyalinus (Rambur), worker termites begin nest construction by building a dome-like structure, or royal chamber, around their much larger and immobile queen (Bruinsma, 1979). Construction of a royal chamber is one aspect of the nest building process that is relatively reproducible and therefore Bruinsma (1979) designed a series of controlled experiments in order to investigate the ways in which pheromones and other factors are involved in the coordination of the construction process.

Bruinsma (1979) observed royal chamber construction after the introduction of a number of worker termites into a perspex box containing a single new cessile queen. Initially, the termites spend time grooming the abdomen of the queen before individuals move away from the queen, picking up a pellet of soil and tracing a meandering path out to a distance 
of about $2-5 \mathrm{~cm}$ where the pellet is placed and secured with faecal cement before returning to the termite queen to groom again. Gradually more and more of the termites repeat this process and in this way a behavioural cycle develops.

Initially, the distribution of deposited soil pellets around the queen appears random but aggregations of material quickly form as termites begin to preferentially approach the existing building sites and add material to them. The distribution of built material becomes concentrated in a few locations where pillars form. Initially the paths taken by a termite before placing a pellet are serpentine and relatively long but, over time, these paths become shorter and straighter, running more directly from the termite queen to a pillar. When the pillars reach a certain height, termites may begin to build horizontally outwards to form lateral extensions termed lamellae. Lamellae from adjacent pillars may eventually join to form arches.

Bruinsma (1979) deduced that the queen termite emits a building pheromone which the worker termites spread over the abdomen while grooming. By diffusing away from the queen's body, this pheromone acts as a template for the building process, effectively defining a deposition zone around the queen by inducing the termites to place soil pellets mainly at a distance where the level of pheromone is within a specific range. Bruinsma (1979) also found that the faecal cement which the termites use to fix soil pellets contains cement pheromone. This pheromone both attracts termites and makes them more likely to deposit a soil pellet. Earlier observations of termite construction by Grassé (1959) led him to theorise that an attractive pheromone infused into the material that termites build with was responsible for the positive feedback effect that results in aggregation of built material and then pillar formation. Grassé (1959) called this process stigmergy.

A two-dimensional mathematical model by Deneubourg (1977) and a two-dimensional cellular automaton by Courtois and Heymans (1991) demonstrated how this positive feedback can occur in a roughly homogeneous distribution of pheromone-infused building material, when material is preferentially moved towards areas with higher levels of the pheromone. Any initial small fluctuations in the distribution of material are amplified resulting in a more peaked distribution. It was hypothesised that these peaks could be identified with the early stages of pillar formation. An extension of the mathematical model by Bonabeau et al. (1998) to include features of Bruinsma's royal chamber experiments showed that the introduction of a pheromone template emitted by the termite queen can restrict the formation of peaks such that they only occur within a certain range of her.

A three-dimensional agent-based version of the extended mathematical model (Ladley and Bullock, 2004, 2005), designed to include more realistic physical constraints on termite movement and building, also showed that cement pheromone could act as a recruiting mechanism to enable the formation of pillars in a deposition zone around an artificial termite queen. Unlike the previous two-dimensional models, in this model it was possible for termites to build truly three-dimensional structures, implying the logical possibility of lamellae construction. However, the model was not able to demonstrate lamellae arising as a result of cementpheromone-mediated positive feedback in the building process.

Further findings by Bruinsma (1979) suggest that the attractive nature of cement pheromone cannot be the only mechanism responsible for pillar formation. The distance over which cement pheromone influences the actions of worker termites was experimentally determined to be approximately $1-1.5 \mathrm{~cm}$, which is less than the distance between the queen and the deposition zone. In addition, experiments revealed that trail pheromone was present in the soil between the queen termite and the deposition zone. Although no specific trail patterns could be identified, Bruinsma (1979) showed that when the termites were prevented from being able to deposit trail pheromone, they were unable to complete construction of the royal chamber and instead built flattened ridges closer to the termite queen, with no evidence of pillar formation. Finally, the observation by Bruinsma (1979) that the paths taken by the worker termites from the queen to the deposition zone become less serpentine as the construction of the royal chamber progresses suggests that they may be leaving trails of pheromone which are influencing the paths taken by other termites and enabling them to more quickly reach active building sites.

To date, no model has incorporated the ability of the termites to deposit and follow trail pheromone while building. Consequently, here, we extend the three-dimensional agentbased model of Ladley and Bullock $(2004,2005)$ in order to investigate the role of trail pheromone in the building process. Our intention is to explore whether the inclusion of trail pheromone can lead to the demonstration of the following observed features of royal chamber construction that have not been modelled to date:

1. Stigmergic recruitment by trail pheromone leading to pillar formation,

2. Characteristic failure of the build process in the absence of trail laying,

3. Formation of lamellae,

4. The appearance of increasingly direct paths from the termite queen to the deposition zone.

In the following sections we first describe the design of the model, before presenting simulation results. We then provide a discussion before the paper concludes.

\section{Methods}

In order to model trail laying and following it is necessary to introduce a simple approximation of the termite behavioural 
cycle observed by Bruinsma so that the termites have distinct behavioural modes during which they are either grooming the queen termite, travelling to the deposition zone, or returning to the queen after building. Depending on model parameters, when en route to the deposition zone or when deciding whether or not to place a piece of building material, termites may be influenced by some combination of the local concentrations of trail pheromone and/or cement pheromone. When returning to the queen after building, termites use the building pheromone in order to orient towards the queen, and lay trail pheromone as they move.

Simulations were carried out using a three-dimensional agent-based model based on that of Ladley and Bullock $(2004,2005)$ in order to incorporate the same logistic constraints and so that comparisons can be made with the output of that model. In the model, space is represented by a threedimensional cubic lattice of size $X \times Y \times Z$, where $Z$ labels the extent of the lattice in the vertical dimension. All locations in the world except edge locations therefore have a set of 26 adjacent neighbours, of which six are cardinal neighbours; two locations are cardinal neighbours if they share a face.

Each location may contain:

1. A single block of one of three types, representing pellets of building material, sections of ground and sections of termite queen. Locations not containing a block are deemed empty.

2. Zero or more worker termites. The total number of termites in the model is denoted $n$ and each follows the behavioural cycle outlined in figure 1 . Termites cannot share locations with blocks.

3. Concentration values for three types of pheromone; building pheromone, cement pheromone and trail pheromone.

Simulations are synchronous and operate for a fixed number of discrete time steps, $t_{\max }$. At each time step, the model updates the behavioural mode and location of each worker termite, adds newly placed blocks of building material as a result of building activity, and updates the concentration values of pheromones at each location.

The locations of blocks of ground and termite queen are fixed at the start of each simulation. Ground blocks are placed in all locations for which $z=1$, and the termite queen is represented as a predetermined configuration of queen blocks placed on top of the ground and centrally with respect to $x$ and $y$. All other locations are initially empty. The initial locations of the worker termites are randomly selected from the set of all empty locations that are cardinal neighbours of locations containing queen blocks. All pheromone values are initially set to 0 .

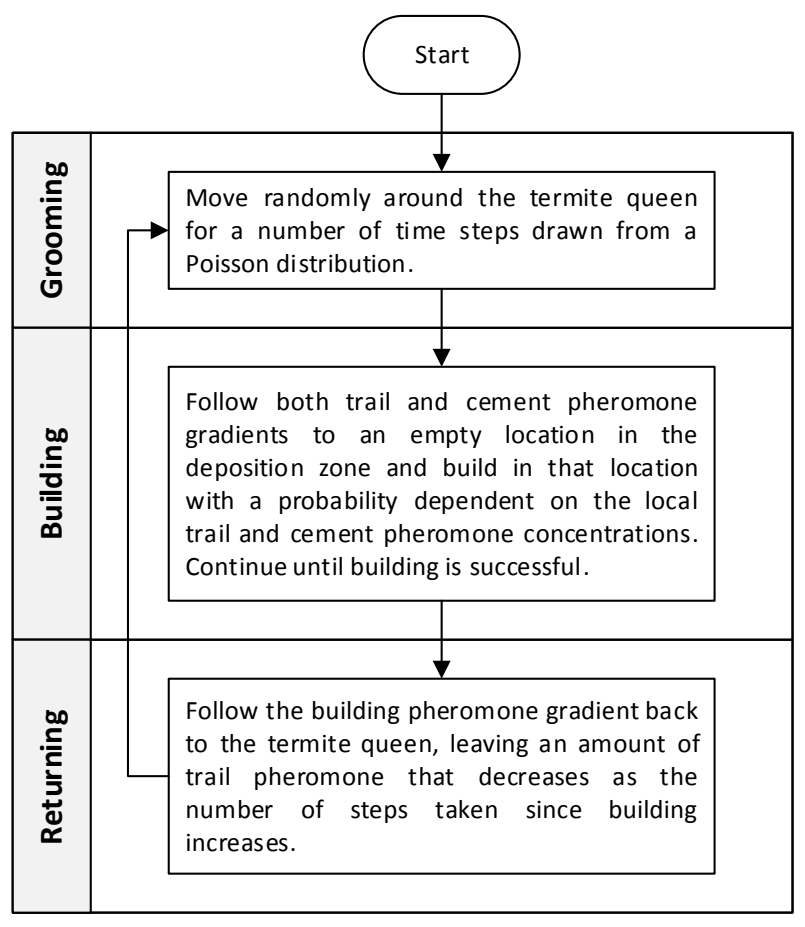

Figure 1: The termite behavioural cycle.

\section{Pheromones}

Each of the pheromone types in the model has a distribution represented by a set of real values defined across all locations. The distributions are parameterised independently and subject to processes of diffusion and evaporation which are applied on every $5^{\text {th }}$ time step.

Pheromone values in all non-edge locations are subject to diffusion between cardinal neighbours. Diffusion between two cardinal neighbours is proportional to the difference in the pheromone values at the two locations and is implemented using the finite volume method (Hirsch, 1988). The amount of pheromone that diffuses from one location with a volume of pheromone $U_{1}$, to a cardinal neighbour with a volume of pheromone $U_{2}$, is given by $\Delta U=-\rho\left(U_{1}-U_{2}\right)$, where $\rho$ is the diffusion coefficient. Pheromone cannot diffuse into locations containing blocks, with the proportion that would have done so remaining in the original location. Pheromone can diffuse into edge locations but is then removed from the simulation so that it cannot diffuse back into non-edge locations. Evaporation of each pheromone type is modelled by multiplying the pheromone value of that type in each location by an evaporation constant, $0<\nu \leq 1$, at each update.

A constant volume of building pheromone is emitted by the termite queen and this is represented by setting the building pheromone value in all locations containing queen blocks to a constant value $\phi_{Q}$ at each time step. Blocks representing pellets of building material contain an initial 
amount of cement pheromone $\phi_{C}$ which is set at the location and time that a block is placed by a termite. The level of cement pheromone remaining at the site of block placement is multiplied at each time step by a factor $0 \leq r \leq 1$, representing the fraction lost through denaturing. Trail pheromone is deposited by termites in each location that they move through on their way back to the queen after placing a pellet of building material (see below).

\section{Termites}

At each time step a termite in location $l$ can in principle move to all locations in the set, $L$, of 26 neighbours surrounding $l$. However, in practice a termite can move only to a subset of these locations; the target set, $K$. The locations that a termite cannot move to are represented by the sets:

1. E: all edge locations,

2. F: all locations containing a block,

3. A: all locations with no cardinal neighbour containing a block (locations deemed to be in mid air),

4. $W$ : empty non-cardinal neighbour locations within $L$ for which all cardinal neighbours that share a side or edge with location $l$ contain a block (these locations are effectively on the other side of a wall),

5. D: a set of the previous locations that a termite has moved through (this set is designed to make trail gradient following more polarised by preventing termites that are following a trail from immediately turning around and walking back in the opposite direction).

Additionally, when in grooming mode termites are subject to a restriction set $R$ which contains all locations that are not cardinal neighbours of queen blocks. When not grooming, $R=\emptyset$. The target set $K$ of locations a termite can move to is therefore given by:

$$
K=L \backslash(E \cup F \cup A \cup R \cup W \cup D)
$$

Once $K$ has been determined, a target location to move to, $k \in K$, is computed depending on the behaviour of the termite. If moving randomly then the probability of moving to each target location $k \in K$ is identical and given by $P_{m}=1 /|K|$. If following a single pheromone type then the probability is given by:

$$
P_{m}(k, l)=\frac{\Delta U(k, l)+\mu}{\sum_{k} \Delta U(k, l)+|K| \mu}
$$

Here, $\Delta U(k, l)$ is any positive difference in pheromone values between $k$ and $l$, given by:

$$
\Delta U(k, l)= \begin{cases}U(k)-U(l), & \text { if }(U(k)-U(l)) \geq 0 \\ 0, & \text { otherwise }\end{cases}
$$

and $\mu$ is a sensitivity parameter which represents the extent to which a termite will move randomly rather than move towards a location with a higher pheromone value. This parameter biases the selection of a target location such that as $\sum_{k} \Delta U(k, l) \rightarrow 0, P_{m}(k, l) \rightarrow 1 /|K|$ and is independently defined for each pheromone type. This ensures that if pheromone differences are large relative to $\mu$ then a termite will be more likely to be influenced by those differences, whereas if they are small then a termite will be more likely to move randomly. Once $P_{m}(k, l)$ has been determined for all $k \in K$, a roulette wheel selection algorithm is used to choose $k$.

If more than one type of pheromone is influencing the movement of a termite then the weighted sum (1 $w) P_{m}^{1}(k, l)+w P_{m}^{2}(k, l)$ is used in the roulette wheel selection algorithm to represent the relative extent to which it is influenced by each type of pheromone $(0 \leq w \leq 1)$.

Grooming Termites begin a simulation by grooming the queen termite. During this time they move randomly around the set of locations that are cardinal neighbours of queen blocks. The time that each termite spends grooming is randomly drawn from a Poisson distribution of expected value $\lambda$. After this time has expired, a termite switches to building behaviour mode. Grooming behaviour ensures that the termites leave the queen to build at different times and from different locations adjacent to the queen.

Building In building behaviour mode it is assumed that each termite has a pellet of building material. The termites search for a location to place the pellet by following both cement and trail pheromone gradients until they reach a location where they could potentially build. This deposition zone defines the overall shape of the royal chamber and is defined as all locations $l$ for which $Q_{\min } \leq Q(l) \leq Q_{\max }$, where $Q(l)$ is the level of building pheromone at location $l$, and $Q_{\min }$ and $Q_{\max }$ are model parameters. Once a termite arrives at a location $l$ in this zone it may place a block of material if at least one of the following is true:

- The cardinal neighbour above $l$ or the cardinal neighbour below $l$ contains a block,

- One of the horizontal cardinal neighbours of $l$ contains a block which has a block either directly above or below it,

- The location $l$ is a horizontal cardinal neighbour of one or two of a set of three neighbouring locations that each contain blocks and which are horizontal cardinal neighbours of each other (so that lateral extensions can only form where there is enough material to support them).

If a termite is able to legally build at location $l$, it does so with a probability $P_{b}(C, T)$ that depends on both the cement pheromone $(C)$ and the trail pheromone $(T)$ concentrations in the location $l$. The form of the build probability 


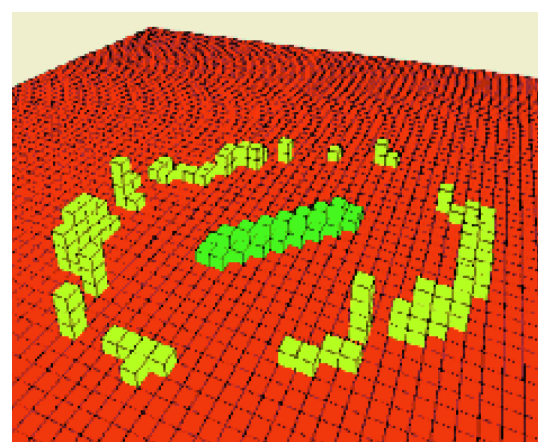

(a) From Ladley and Bullock (2004).

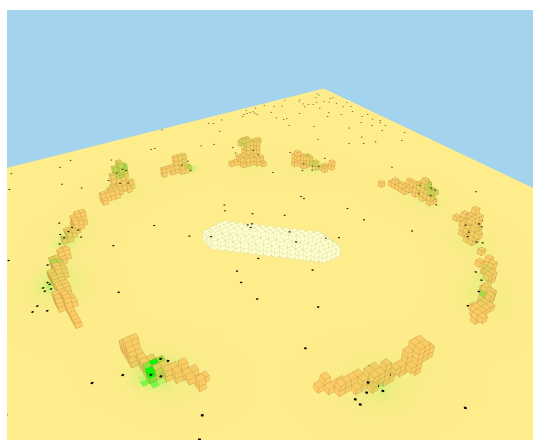

(d) 150 time steps.

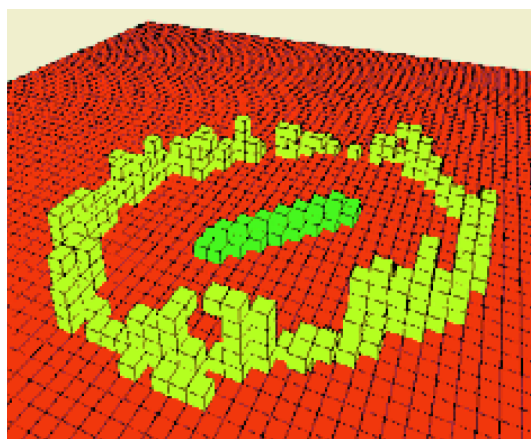

(b) From Ladley and Bullock (2004).

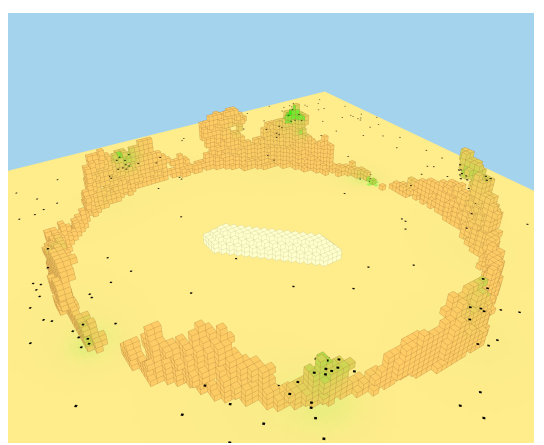

(e) 600 time steps.

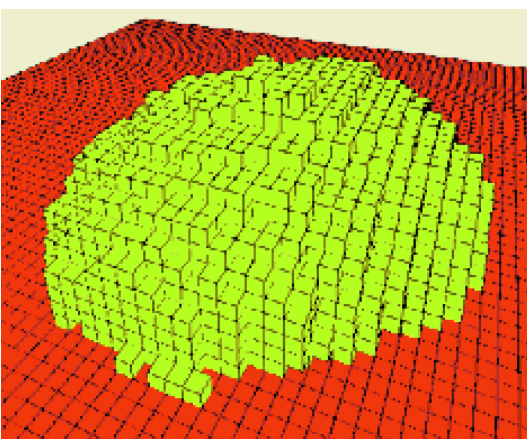

(c) From Ladley and Bullock (2004)

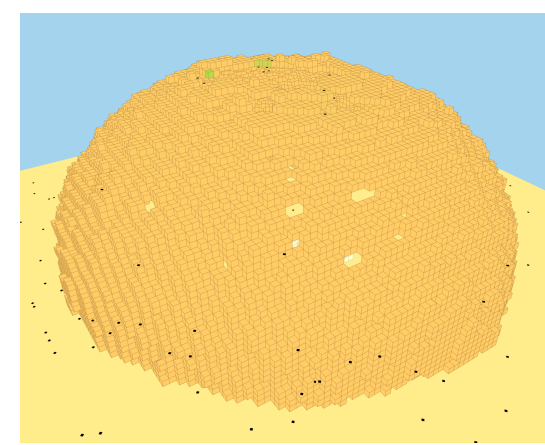

(f) 10000 time steps.

Figure 2: Replication of, and comparison with, a royal chamber construction simulation by Ladley and Bullock (2004). Termites are represented by black diamonds and cement pheromone is indicated by green shading.

is a combination of two logistic functions, each representing the influence of a pheromone type. It is given by:

$$
P_{b}(C, T)=\left(P_{\min }+\frac{P_{\max }-P_{\min }}{1+e^{-k_{1}\left(C-C_{\max } / 2\right)}}\right) f(T)
$$

where:

$$
f(T)=\left(1-\frac{1}{1+e^{-k_{2}\left(T-T_{\max } / 2\right)}}\right)
$$

The function, $f(T)$, allows the presence of trail pheromone to inhibit building behaviour, modulating the probability of building such that $P(C, T) \rightarrow 0$ as $T \rightarrow$ $T_{\max }$ for all $C$. This function is motivated by observations that termites tend not to build where trail pheromone concentration is high (Bruinsma, 1979). $P_{\min }$ and $P_{\max }$ are the minimum and maximum build probabilities in the absence of trail pheromone, and obtain when the levels of cement pheromone are 0 or $C_{\max }$, respectively. Parameters $k_{1}$ and $k_{2}$ define the steepness of the logistic functions.

Returning After placing a pellet of building material a termite returns to the queen by following the building pheromone gradient. During this journey the termite deposits trail pheromone in each location that it moves through. The amount of trail pheromone that a termite leaves in each location decreases linearly with time so that a positive gradient which other termites can follow when finding a building location is created as simply as possible. The amount is given by:

$$
T(t)=\phi_{T} \frac{\max \left(L_{T}-t, 0\right)}{L_{T}-t} .
$$

where $\phi_{T}$ is an initial value of trail pheromone, $t$ is the number of time steps since the termite placed a block and $L_{T}$ is a parameter defining the length of time over which each termite is able to leave a trail.

\section{Results}

All simulations were carried out in a world of size $120 \times$ $120 \times 60$ locations. This is larger than the world size used by Ladley and Bullock $(2004,2005)$ and other parameters were adjusted accordingly so that the simulations were effectively operating at a higher resolution. The configuration of queen blocks making up the termite queen was modified so that it was twice the size of the original in all dimensions. The purpose of increasing the resolution of the simulations was to improve the separation between, and definition of, pheromone trails.

We first replicate the creation of a royal chamber in the manner of Ladley and Bullock (2004, 2005). In this earlier 


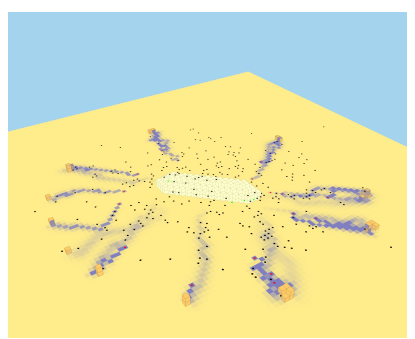

(a) 150 time steps.

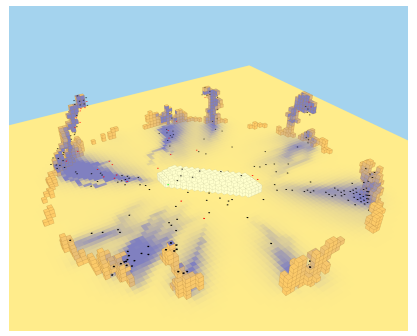

(c) 600 time steps.

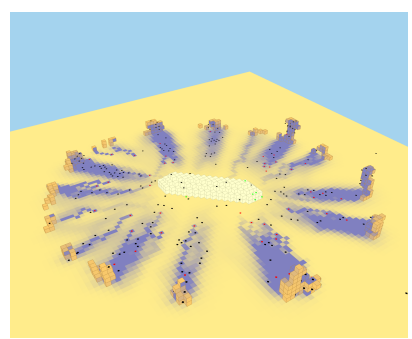

(b) 300 time steps.

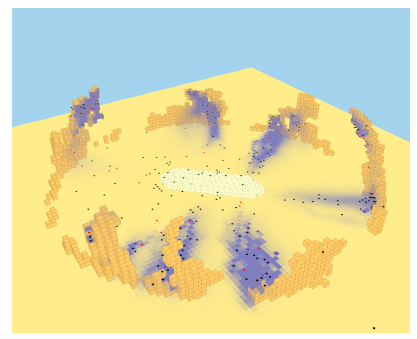

(d) 1200 time steps.
Figure 3: Construction of a royal chamber using building, cement and trail pheromones. Trail pheromone is indicated by purple shading.

model the worker termites permanently exhibit only building behaviour and follow only cement pheromone gradients to the deposition zone. Blocks of building material are placed with a fixed probability and after building each termite is removed from the world and replaced at random. Both queen and cement pheromones have the same parameters in this model, $\left(\phi_{Q}=\phi_{C}=400, \rho_{Q}=\rho_{C}=1 / 7\right.$, $\left.\nu_{Q}=\nu_{C}=0.01\right)$ and $r=0.5$. The number of termites is $n=400$ and the fixed build probability is $P_{b}=0.1$. $Q_{\min }$ and $Q_{\max }$ are 0.1 and 0.2 respectively and the sensitivity parameter for following cement pheromone is set to $\mu_{C}=0.005$. These parameters were chosen so that the replicated model output was visually a good match to the earlier model. We also follow Ladley and Bullock (2004, 2005) in allowing simulations to run for a number of time steps before introducing worker termites in order to establish a stable building pheromone template defining the deposition zone.

The new model is able to successfully replicate the output from the earlier model at higher resolution (figure 2). Initially, building is concentrated at a few sites. Mound-like pillars form at these sites, before low walls between them are constructed and eventually a dome is completed.

To explore the combined influence of building, cement and trail pheromones, and in keeping with the observations of Bruinsma (1979), the cement pheromone parameters in the new model were chosen so that the attractive influence of the pheromone from the deposition zone does not extend as far as the body of the termite queen. Trail following by the termites therefore becomes important in order to direct them

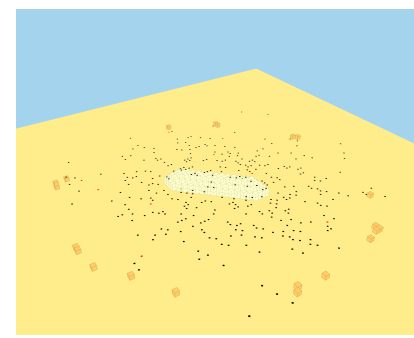

(a) 150 time steps.

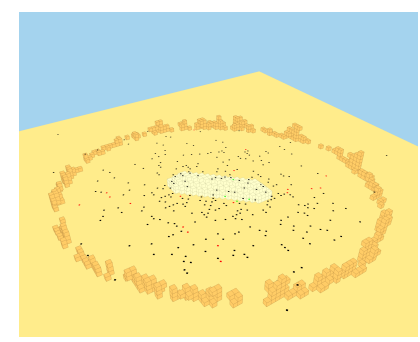

(c) 600 time steps.

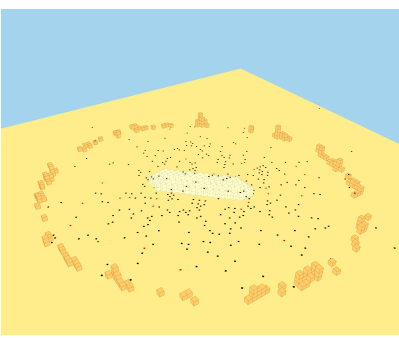

(b) 300 time steps.

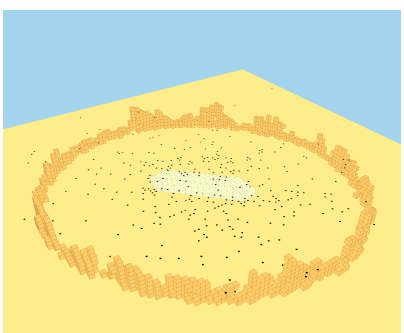

(d) 1200 time steps.
Figure 4: Attempted construction of a royal chamber when termites are prevented from leaving trail pheromone. All parameters are the same as figure 3 .

to sites in the building zone that already have building material and thereby achieve a significant stigmergic effect. The value used for for $w$ was 0.001 so that the total probability of a termite moving to a location $k$ is much more influenced by cement pheromone if the levels of both pheromones are comparable.

The number of time steps subsequent to placing a block for which termites can leave trail pheromone, $L_{T}$, was set to 25 , which is the approximate number of locations from the foot of the deposition zone to the termite queen, to ensure that the amount of trail pheromone deposited approaches 0 near the queen.

Pheromone parameters were $\phi_{Q}=\phi_{C}=\phi_{T}=400$, $\rho_{Q}=1 / 7, \rho_{C}=\rho_{T}=0.017, \nu_{Q}=0.01, \nu_{C}=0.1, \nu_{T}=$ 0.01 and $r=0.5$. Values selected for the build probability parameters were $P_{\min }=0.1, P_{\max }=0.3, k_{1}=k_{2}=0.1$, $C_{\max }=\phi_{C} / 2$ and $T_{\max }=\phi_{T} / 2$.

Example output is shown in figure 3. The pheromone trails created by the termites are clearly visible and the model retains the capacity to produce peaks and pillars that are similar to those shown in the model of Ladley and Bullock $(2004,2005)$. In addition, there is visible evidence of lamellae formation and at later stages of construction the termites are following the trails to the deposition zone, whereas at earlier stages their movement is more random. These features are in keeping with the observations of Bruinsma (1979).

The experiment of Bruinsma (1979) in which the termites are prevented from depositing trail pheromone is recreated in the model and the result is shown in figure 4 . In this 
口Cement Pheromone $\quad \square$ Random Movement

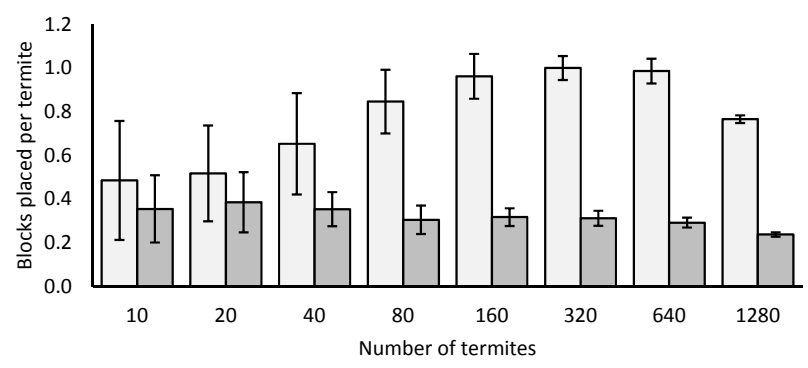

Figure 5: Recruitment curves for the replicated model of Ladley and Bullock (2004, 2005) with a world size matching the earlier model. Each value is a mean over 20 simulations, with each simulation running for 200 time steps. Error bars represent + /- one standard deviation.

QTrails $\square$ No Trails

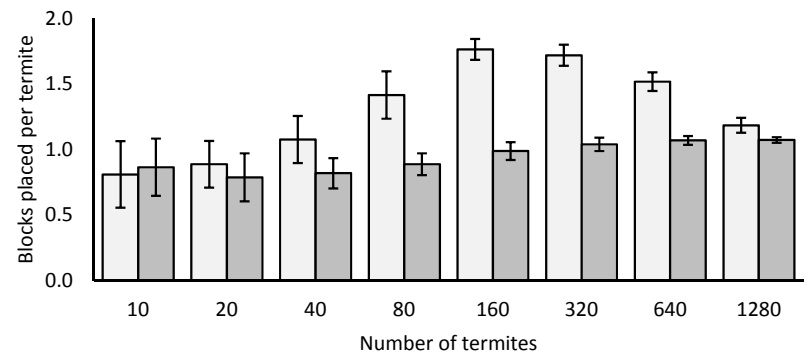

Figure 6: Recruitment curves for the new model. Each value is a mean over 20 simulations, with each simulation running for 600 time steps. Error bars represent +/- one standard deviation.

case there is visible evidence that the termite building has slowed considerably and there is no significant pillar formation, although there is still some visible peak formation at later times. The attractive range of the cement pheromone is not large enough to allow the termites to follow it from the termite queen to the deposition zone but it still has a local stigmergic effect, allowing the formation of some small vertical enhancements. This demonstrates how pillar formation might be disrupted by preventing the deposition of trail pheromone.

To demonstrate a qualitative correspondence between their model and the observations of Bruinsma (1979), Ladley and Bullock (2005) produced a graph of the mean number of blocks placed per termite after a fixed number of time steps had elapsed, for different termite colony sizes (i.e., different values of $n$ ). Bruinsma (1979) produced a similar graph for worker termites to show that the build rate per termite is low for small colony sizes since recruitment to building sites is not efficient at low population density, but that the stigmergic feedback effect increases this build rate as the size of the colony grows until a saturation level is reached, when there is interference between termites com- peting to build. Equivalent results for the replicated model are shown in figure 5.

For comparison, figure 5 also shows a graph for scenarios in which there is no cement pheromone attraction. In this case, the number of blocks placed per termite decreases slightly with increasing colony size, which we take as an indication that stigmergic activity is not present.

Figure 6 shows equivalent results for the new model. When trail pheromone deposition is allowed the graph demonstrates that a stigmergic feedback effect is present, as in the earlier model and observations. When trail pheromone deposition is prevented the blocks placed per termite appears to be more constant across colony size but still increases slightly and does not follow the same pattern as the random scenario in figure 5. This is consistent with the much reduced stigmergic effect of more localised cement pheromone attraction shown in figure 4.

\section{Discussion}

The simulations demonstrate behaviour that agrees qualitatively with observations of termite royal chamber construction and suggest a plausible mechanism through which trail pheromone may be involved in such construction.

The algorithms governing pheromone following, build probability and trail deposition are not known to be ideal and have been chosen for simplicity or plausibility. We do not know exactly how or when the trails of termites building a royal chamber are laid, if they need to be reinforced in both directions or if perhaps the strength of the pheromone trail laid is dependent on the amount of cement pheromone detected before building, for example. Implementing the model on a three-dimensional cubic lattice is also not ideal because the anisotropic nature of the neighbour distribution makes the design of a trail following algorithm difficult. The introduction of the restriction preventing termites from walking back over their last few previous locations was an attempt to alleviate this difficulty.

Furthermore, there are several additional factors involved in termite construction that have not been included in the model but which may influence building behaviour, such as gravity, temperature or humidity. Termites are also known to be influenced by tactile stimuli, so that sharp angles and existing obstacles incite building activity (Stuart, 1967; Bruinsma, 1979).

One explanation for why the model is able to show narrower pillar formation and lateral growth and the earlier model of Ladley and Bullock $(2004,2005)$ was not is the fact that, as well as directing termites to the deposition zone, trail pheromone also supresses building activity. This means that after some pellets of building material have been placed at lower elevations there is enough trail pheromone at these elevations to reduce the probability of further building. The termites therefore effectively have to move upwards to find suitable build locations, and once at higher elevations they 
are not prevented from building laterally.

However, once enough termites are building at higher elevations, the concentration of trail pheromone at lower elevations becomes insufficient to prevent building and additional construction occurs from the ground up once more. This effect is exacerbated by the termites effectively getting stuck for a number of time steps at higher elevations as they have no concept of 'down'. During this time the trails weaken and subsequent building becomes more spread out.

The next step is to investigate objective, quantitative measures of the model outcomes and processes in order that it can be automatically determined whether or not a model run is successful. These quantitative measures will be necessary in order to use a sensitivity analysis or statistical emulators to determine the influence of model parameters. Methods for quantifying the model output could involve the use of autocorrelation functions, entropy measures, mathematical morphology (Serra, 1982) or measures of clustering, for example. Given the shape of the royal chamber and the binary nature of the block distribution in three dimensions (locations either have a block or do not), the application of such methods presents a challenge.

Some simpler initial analysis of the effects of the model parameters on factors such as the change in the distribution of build probabilities with time step or the minimum rate of block placement required to maintain a desired attractive radius of cement pheromone may help to identify key parameter ranges.

\section{Conclusion}

A new three-dimensional agent-based model has been established that is able to reproduce earlier work by Ladley and Bullock (2004, 2005). Moreover, by extending the model to include a behavioural cycle documented by Bruinsma (1979), we have been able to qualitatively demonstrate that pillar formation can also occur when the movement and building behaviours of termites are influenced by a combination of both cement and trail pheromones. This three-pheromone model additionally shows lateral growth from pillars (lamellae), movement from the termite queen to the deposition zone that becomes more direct as trails form, and a distribution of building material that has significantly suppressed pillar formation when trail pheromone deposition is prevented. These features are consistent with termite royal chamber construction (Bruinsma, 1979) and have not been simulated in earlier models (Deneubourg, 1977; Courtois and Heymans, 1991; Bonabeau et al., 1998; Ladley and Bullock, 2004, 2005).

The next step is the quantitative analysis of the model parameters, algorithms and output in order to identify redundancies in the parameter space, and regimes or critical points in the dynamics of the model and to distinguish between more or less successful simulations of royal chamber construction.

\section{Acknowledgements}

This work was supported by an EPSRC Doctoral Training Centre grant (EP/G03690X/1).

\section{References}

Bonabeau, E., Theraulaz, G., Deneubourg, J.-L., Franks, N. R. Rafelsberger, O., Joly, J.-L., and Blanco, S. (1998). A model for the emergence of pillars, walls and royal chambers in termite nests. Philosophical Transactions of the Royal Society of London B, 353(1375):1561-1576.

Bruinsma, O. H. (1979). An analysis of building behaviour of the termite macrotermes subhyalinus (rambur). $\mathrm{PhD}$ thesis, Wageningen University.

Courtois, P.-J. and Heymans, F. (1991). A simulation of the construction process of a termite nest. Journal of Theoretical Biology, 153(4):469-475.

Deneubourg, J.-L. (1977). Application de l'ordre par fluctuations a la description de certaines étapes de la construction du nid chez les termites. Insectes Sociaux, 24(2):117-130.

Doursat, R., Sayama, H., and Michel, O., editors (2012). Morphogenetic Engineering: Toward Programmable Complex Systems. Springer.

Grassé, P.-P. (1959). La reconstruction du nid et les coordinations inter-individuelles chez bellicositermes natalensis et cubitermes sp. la théorie de la stigmergie: Essai d'interprétation du comportement des termites constructeurs. Insectes Sociaux, $6(1): 41-80$

Hirsch, C. (1988). Numerical Computation of Internal and External Flows Volume 1: Fundamentals of Numerical Discretization. Wiley-Interscience.

Howse, P. E. (1970). Termites: A Study in Social Behaviour. Hutchinson \& Co Ltd.

Ladley, D. and Bullock, S. (2004). Logistic constraints on 3d termite construction. In Ant Colony Optimization and Swarm Intelligence (Proceedings of ANTS 2004), pages 178-189. Springer.

Ladley, D. and Bullock, S. (2005). The role of logistic constraints in termite construction of chambers and tunnels. Journal of Theoretical Biology, 234(4):551-564.

Leuthold, R. H., Bruinsma, O. H., and van Huis, A. (1976). Optical and pheromonal orientation and memory for homing distance in the harvester termite hodotermes mossambicus (hagen). Behavioral Ecology and Sociobiology, 1(2):127-139.

Serra, J., editor (1982). Image Analysis and Mathematical Morphology. Academic Press, Inc.

Stuart, A. M. (1967). Alarm, defense, and construction behavior relationships in termites (isoptera). Science, 156(3778):11231125.

Stuart, A. M. (1970). The role of chemicals in termite communication. In Advances in chemoreception volume 1: Communication by chemical signals, pages 79-106. Appleton-CenturyCrofts.

Wilson, E. O. (1971). The Insect Societies. Harvard University Press. 